\title{
SPELLING TEST FOR LEARNERS WITH DYSLEXIA LEARNING GERMAN AS A SECOND FOREIGN LANGUAGE
}

\author{
Sofia P. Tsakalidou ${ }^{\mathrm{i}}$ \\ Aristotle University of Thessaloniki, \\ Greece \\ orcid.org/0000-0001-5500-1586
}

\begin{abstract}
:
Spelling is a basic skill as well as means of acquiring knowledge over the school years. Moreover, it is a complex cognitive process, which can be challenging for learners with specific learning difficulties (dyslexia) in their mother language as well as when learning a foreign language. A challenge, that foreign language teachers face in everyday practice, is being able to respond to the needs of all learners within the mainstream classroom in primary education, including learners with dyslexia. Foreign language teachers need to become equipped with further knowledge and skills, so that they are able to identify these difficulties and teach learners with dyslexia more effectively in an inclusive way (Tsakalidou, Koufokotsiou, \& Gaganis, in press; Tsakalidou, 2021, 2020, in press). In the present study we examined the spelling difficulties of learners with dyslexia, when learning German as a second foreign language. The aim of the study was twofold: (a) the comparative analysis of the spelling difficulties across two populations (95 learners between 10 and 11 years, twenty of who had dyslexia) and (b) the evaluation of the effectiveness of spelling intervention in respect to learners with dyslexia. In this article we will describe the Spelling Test constructed and adjusted for the doctoral research, which was undertaken in order to record the spelling difficulties that the learners with dyslexia face, when learning German as a second foreign language in primary education. This test was based on a standardized Spelling Test in the Greek language (Mouzaki, Protopapas, Sideridis, \& Simos, 2010). As far as the Spelling Test, we constructed is concerned, satisfactory construct validity and internal reliability were demonstrated, as the Cronbach's alpha coefficient was .944 and revealed a high degree of internal consistency of the instrument.
\end{abstract}

Keywords: orthographic skill, specific learning difficulties, dyslexia, German as a foreign language, primary education

i Correspondence: email stsakalidou@del.auth.gr 


\section{Introduction}

Spelling is a basic skill as well as means of acquiring knowledge over the school years. Furthermore, it is a complex cognitive process, which can be challenging for learners with specific learning difficulties (dyslexia). The foreign language teachers face the everyday challenge of teaching learners with dyslexia within the mainstream classroom in primary education (Tsakalidou, Koufokotsiou, \& Gaganis, in press; Tsakalidou, 2021, 2020, in press). It would be of great importance, if teachers had a tool in their hands, in order to be able to identify and record the spelling performance of learners and adjust their teaching to the needs of all learners (Bahr, Silliman, \& Berninger, 2009).

\section{Literature Review}

After a detailed literature review, we reached the conclusion, that there are very few publications about dyslexia and foreign language acquisition and teaching in the Greek bibliography (Andreou \& Baseki, 2012; Reraki, 2020; Sougari \& Mavroudi, 2019; Talli, 2010; Tsakalidou, Koufokotsiou, \& Gaganis, in press; Tsakalidou, 2021, in press) and this doctoral thesis (Tsakalidou, 2020) is the first effort to approach this subject in detail, as far as the German language is concerned. Also, the spelling tests constructed for native speakers learning the German language were not considered appropriate in this case, as the age and reference level were not the same for learners of German as a second foreign language (Landerl, Wimmer, \& Moser, 1997; Stock \& Schneider, 2008).

\section{Material and Methods}

The present research is part of the author's doctoral thesis (Tsakalidou, 2020). In the present study we examined the spelling difficulties of learners with dyslexia, when learning German as a second foreign language. The aim of the study was twofold: (a) the comparative analysis of the spelling difficulties across two populations (95 learners between 10 and 11 years, twenty of who had dyslexia) and (b) the evaluation of spelling intervention effectiveness in respect to learners with dyslexia. The sample consisted of 12 male and 8 female learners with dyslexia, as well as 35 male and 40 female learners without dyslexia (Chart 1).

A basic tool of the research process was the Spelling Test. Our study was conducted in three phases. During Phase 1 we attempted to compare the common spelling errors and characteristics across the two populations (learners with and without dyslexia). During Phase 2 we attempted to plan, organize and assess the intervention in the GFL lesson. Finally, during Phase 3 we recorded the spelling performance pre and post intervention across the two populations. The Spelling Test was used in Phases 1 and 3 (pre- and post-performance) and during Phase 2 various interventions were implemented in order to develop the orthographic skill of learners with and without dyslexia. 


\section{Chart 1: Research sample}

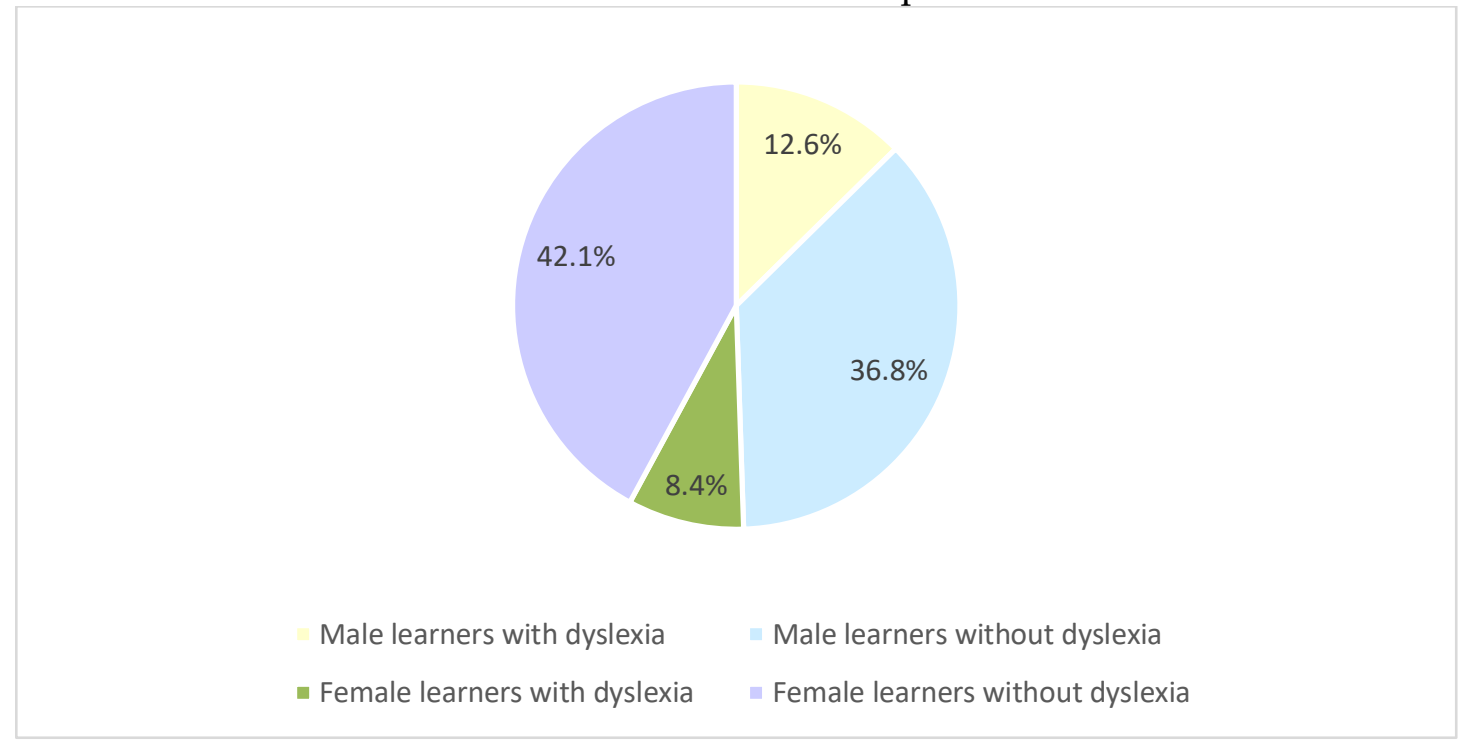

The Spelling Test was a basic tool used to record specific difficulties of learners in respect to orthographic skill in their mother language (Greek) and in the second foreign language (German), in order to plan and organize the intervention and differentiate instruction during Phase 2 of our research. It was administered to all learners with and without dyslexia.

As far as the Spelling Test is concerned, satisfactory construct validity and internal reliability were demonstrated, as the Cronbach's alpha coefficient was very high and revealed a high degree of internal consistency of the instrument, namely .944.

The process followed, in order to create the Spelling Test, is described in detail. We analysed the three books, which had been approved for teaching German as a second foreign language in primary schools (during the years 2015-2017) (Bovermann, Georgiakaki \& Zschärlich, 2013; Jenkins-Krumm \& Clement, 2014; Kounalaki, Delikeisoglou, Brodowski-Frogaki, Koutentaki, \& Papandreou, 2011) and constructed the Spelling Test based on a standardized Spelling Test in the Greek language (mother language of the learners) (Mouzaki, Protopapas, Sideridis, \& Simos, 2010).

All the words from the first two chapters of each book were recorded according to two criteria, namely the number of syllables (monosyllabic, disyllabic, trisyllabic and polysyllabic words) (Tables 1-10) and the graphemes contained in the words (ei, eu, ie, au, ä, ö, ü, sch, sp, st, ch, -er, -th-, -g, -d, chs, ng) (Tables 11-13). 
Table 1: List of monosyllabic words in Paul, Lisa \& Co. 1

\begin{tabular}{|c|c|c|c|c|}
\hline acht, $7^{\mathrm{ii}}$ & die, 18 & hier, 18 & noch, 34 & vier, 7 \\
\hline auch, 12 & doch, 18 & ich, 11 & schau, 18 & von, 21 \\
\hline aus, 23 & drei, 7 & ihr, 28 & schon, 21 & wann, 32 \\
\hline Ball, 19 & $\mathrm{du}, 11$ & ist, 10 & Schuh, 6 & was, 13 \\
\hline Bild, 11 & echt, 18 & ja, 6 & sechs, 7 & wer, 11 \\
\hline bin, 10 & ein, 14 & Jahr, 22 & seid, 10 & wie, 11 \\
\hline bist, 10 & eins, 7 & kommt, 18 & sie, 24 & wir, 27 \\
\hline blöd, 18 & elf, 21 & Lust, 28 & spät, 34 & wo, 34 \\
\hline Buch, 18 & er, 23 & machst, 14 & Spiel, 18 & zehn, 7 \\
\hline Bus, 6 & erst, 21 & macht, 14 & spielst, 11 & Zeit, 28 \\
\hline dann, 34 & Film, 18 & mal, 18 & stopp, 34 & zwei, 7 \\
\hline das, 18 & Frau, 10 & nein, 6 & süß, 23 & zwölf, 21 \\
\hline denn, 11 & fünf, 7 & neu, 11 & toll, 12 & Sum: 72 words \\
\hline der, 18 & hat, 20 & neun, 7 & Uhr, 33 & \\
\hline Deutsch, 5 & heißt, 11 & nicht, 31 & um, 32 & \\
\hline
\end{tabular}

Table 2: List of disyllabic words in Paul, Lisa \& Co. 1

\begin{tabular}{|c|c|c|c|c|}
\hline achtzehn, 21 & gute, 11 & Klavier, 29 & neunzehn, 21 & skaten, 29 \\
\hline Auto, 6 & guten, 11 & lesen, 31 & Note, 6 & Socke, 6 \\
\hline bitte, 12 & habe, 28 & lustig, 18 & Physik, 6 & spiele, 11 \\
\hline Brille, 18 & hallo, 11 & mache, 14 & raten, 32 & suche, 12 \\
\hline Bruder, 21 & heiße, 11 & möchte, 30 & reiten, 31 & super, 6 \\
\hline dreizehn, 21 & heute, 28 & möchten, 30 & sammelst, 18 & tanzen, 31 \\
\hline einen, 14 & Hobby, 12 & möchtest, 30 & sammelt, 18 & Tennis, 11 \\
\hline fertig, 34 & Karte, 5 & Morgen, 11 & schwimmen, 31 & vierzehn, 21 \\
\hline Foto, 14 & keine, 28 & Musik, 6 & sechzehn, 21 & woher, 23 \\
\hline fünfzehn, 21 & Kino, 18 & Müsli, 6 & sieben, 7 & zwanzig, 21 \\
\hline Fußball, 6 & Klasse, 10 & Name, 6 & siebzehn, 21 & Sum: 54 words \\
\hline
\end{tabular}

Table 3: List of trisyllabic and polysyllabic words in Paul, Lisa \& Co. 1

\begin{tabular}{|l|l|l|}
\hline \multicolumn{2}{|c|}{ Trisyllabic words } & \multicolumn{1}{c|}{ Polysyllabic words } \\
\hline Adresse, 6 & Nachmittag, 28 & Hausaufgaben, 14 \\
\hline Alphabet, 7 & Playstation, 18 & interessant, 18 \\
\hline Basketball, 11 & reinkommen, 18 & Marmelade, 6 \\
\hline Computer, 19 & Stadion, 6 & Mathematik, 6 \\
\hline faulenzen, 31 & Volleyball, 9 & Modellauto, 18 \\
\hline fernsehen, 29 & Sum: 11 words & Schokolade, 6 \\
\hline & & Sum: 6 words \\
\hline
\end{tabular}

\footnotetext{
ii The number next to the word is the page number, on which we first see the specific word in the coursebook.
} 
Sofia P. Tsakalidou

SPELLING TEST FOR LEARNERS WITH DYSLEXIA

LEARNING GERMAN AS A SECOND FOREIGN LANGUAGE

Table 4: List of monosyllabic words in Luftballons

\begin{tabular}{|c|c|c|c|c|}
\hline $\mathrm{ABC}, 14$ & die, 9 & ich, 8 & Nacht, 10 & was, 18 \\
\hline am, 11 & $\mathrm{du}, 8$ & ihr, 21 & nein, 12 & wer, 8 \\
\hline auch, 21 & Eis, 12 & in, 21 & nennt, 23 & wie, 8 \\
\hline auf, 10 & Fan, 21 & ist, 20 & nicht, 12 & wir, 21 \\
\hline Ball, 16 & Frau, 9 & ja, 13 & nur, 11 & Wurm, 17 \\
\hline bei, 11 & fünf, 18 & Kind, 16 & Schatz, 23 & Zoo, 13 \\
\hline bin, 8 & gern, 21 & magst, 21 & so, 11 & Zug, 17 \\
\hline bist, 8 & groß, 13 & man, 10 & Tag, 10 & zwei, 10 \\
\hline Clown, 13 & grün, 18 & März, 18 & Tschüss, 9 & zwölf, 18 \\
\hline das, 8 & Gruß, 10 & Maus, 16 & Uhr, 17 & Sum: 61 words \\
\hline dein, 20 & grüßst, 11 & mein, 20 & und, 8 & \\
\hline der, 8 & Heft, 16 & mich, 23 & uns, 11 & \\
\hline Deutsch, 10 & heißt, 8 & Milch, 12 & von, 21 & \\
\hline
\end{tabular}

Table 5: List of disyllabic words in Luftballons

\begin{tabular}{|c|c|c|c|c|}
\hline Abend, 10 & Globus, 16 & Klasse, 9 & müde, 11 & Seite, 10 \\
\hline alle, 13 & gute, 10 & können, 19 & München, 18 & sicher, 21 \\
\hline Bärchen, 23 & guten, 10 & Lampe, 16 & Name, 17 & Sonne, 17 \\
\hline Beispiel, 10 & Hallo, 8 & Liebe, 12 & Namen, 20 & spiele, 21 \\
\hline bilden, 13 & heiße, 8 & Liebling, 23 & nennen, 23 & Tafel, 17 \\
\hline Buchstabe, 11 & heißen, 13 & lila, 13 & Nomen, 13 & Tennis, 21 \\
\hline Computer, 16 & Hobby, 12 & Liste, 23 & Oma, 17 & Umlaut, 14 \\
\hline deutsche, 20 & hören, 10 & Mama, 23 & Party, 13 & unten, 23 \\
\hline Deutschland, 16 & Hotel, 12 & Mäuschen, 23 & passen, 12 & Vogel, 17 \\
\hline fehlen, 11 & Idee, 16 & meine, 23 & passend, 16 & welch-, 10 \\
\hline Fenster, 16 & jed-, 16 & Menü, 18 & Pinsel, 17 & Zettel, 13 \\
\hline finden, 10 & Juni, 16 & mögen, 20 & Quelle, 17 & Sum: 67 words \\
\hline Fußball, 21 & Kästchen, 16 & Morgen, 10 & sage, 11 & \\
\hline ganzen, 11 & Kinder, 9 & morgens, 11 & schreiben, 11 & \\
\hline
\end{tabular}

Table 6: List of trisyllabic words in Luftballons

\begin{tabular}{|c|c|c|c|c|}
\hline Alphabet, 14 & Dänemark, 18 & fehlend-, 19 & sortieren, 23 & vorstellen, 9 \\
\hline anderen, 21 & einkreisen, 10 & griechische, 20 & Spitzname, 23 & Wiedersehen, 9 \\
\hline anschauen, 10 & Elefant, 13 & Gute Nacht, 10 & Sportlehrer, 21 & Xylophon, 17 \\
\hline antworten, 10 & Engelchen, 23 & Guten Tag, 10 & Telefon, 13 & Ypsilon, 17 \\
\hline Basketball, 13 & ergänzen, 19 & häufige, 23 & Turnhalle, 21 & zuhören, 12 \\
\hline beginnen, 13 & erkennen, 19 & jeweilig-, 12 & verbinden, 18 & Sum: 37 words \\
\hline Buchstabe, 15 & Europa, 13 & Österreich, 18 & verstecken, 22 & \\
\hline Computer, 16 & Februar, 13 & Prinzessin, 23 & Vorname, 23 & \\
\hline
\end{tabular}

Table 7: List of polysyllabic words in Luftballons

\begin{tabular}{|l|l|}
\hline Anfangsbuchstabe, 13 & Marmelade, 13 \\
\hline Bildworträtsel, 13 & Namensliste, 20 \\
\hline Biologie, 12 & nummerieren 8 \\
\hline buchstabieren, 22 & Radiergummi, 17 \\
\hline Familienname, 23 & unterstreichen 13 \\
\hline Guten Morgen, 10 & wiederholen 12 \\
\hline Kosename, 23 & Sum: 13 words \\
\hline
\end{tabular}


Sofia P. Tsakalidou

SPELLING TEST FOR LEARNERS WITH DYSLEXIA

LEARNING GERMAN AS A SECOND FOREIGN LANGUAGE

Table 8: List of monosyllabic words in Wir Kids 1

\begin{tabular}{|c|c|c|c|c|}
\hline acht, 7 & die, 7 & gut, 11 & nett, 19 & Tschüs, 9 \\
\hline alt, 9 & doof, 19 & hast, 18 & neun, 7 & und, 6 \\
\hline auf, 9 & dran, 8 & hat, 18 & nicht, 21 & vier, 7 \\
\hline aus, 11 & drei, 7 & heißt, 6 & null, 7 & von, 14 \\
\hline bin, 6 & $\mathrm{du}, 6$ & Herr, 7 & sag, 22 & wer, 6 \\
\hline bist, 6 & eins, 7 & ich, 6 & Schweiz, 17 & wie, 6 \\
\hline blöd, 19 & elf, 7 & ist, 6 & sechs, 7 & Wien, 17 \\
\hline da, 11 & er, 12 & ja, 7 & sie, 10 & Zahl, 8 \\
\hline das, 6 & Frau, 7 & komm, 8 & sind, 10 & zehn, 7 \\
\hline dein, 13 & Freund, 14 & mal, 22 & Sohn, 21 & zwei, 7 \\
\hline der, 7 & fünf, 7 & mein, 12 & Spiel, 8 & zwölf, 7 \\
\hline deutsch, 17 & für, 7 & Mensch, 8 & streng, 19 & Sum: 63 words \\
\hline dich, 6 & grüß, 6 & nein, 7 & Tag, 11 & \\
\hline
\end{tabular}

Table 9: List of disyllabic words in Wir Kids 1

\begin{tabular}{|c|c|c|c|c|}
\hline achtzehn, 8 & Freundin, 14 & Junge, 7 & Oma, 13 & Stuttgart, 11 \\
\hline achtzig, 20 & freundlich, 19 & Jungen, 7 & Onkel, 14 & Tage, 11 \\
\hline Alter, 10 & fünfzehn, 8 & keine, 21 & Opa, 13 & \begin{tabular}{|l|} 
Tante, 14 \\
\end{tabular} \\
\hline bitte, 21 & fünfzig, 20 & Kinder, 21 & Papa, 13 & Tochter, 21 \\
\hline Bruder, 12 & Grüße, 11 & Leute, 11 & Schwester, 13 & Vater, 12 \\
\hline Brüder, 18 & grüßen, 6 & lustig, 19 & Schwestern, 18 & Vati, 13 \\
\hline Danke, 22 & guten, 11 & Mädchen, 7 & sechzehn, 8 & \begin{tabular}{|l|} 
viele, 19 \\
\end{tabular} \\
\hline deine, 13 & habe, 18 & meine, 12 & sechzig, 20 & vierzehn, 8 \\
\hline dreißig, 20 & haben, 21 & Mutter, 12 & sieben, 7 & vierzig, 20 \\
\hline dreizehn, 8 & Hallo, 6 & Mutti, 13 & siebzehn, 8 & Würfel, 8 \\
\hline einen, 18 & heiße, 6 & Name, 7 & siebzig, 20 & Zahlen, 7 \\
\hline Eltern, 14 & heißen, 6 & Namen, 7 & singen, 11 & zwanzig, 8 \\
\hline Foto, 7 & Herren, 7 & neunzehn, 8 & Spiele, 8 & Sum: 72 words \\
\hline Fotos, 7 & hundert, 20 & neunzig, 20 & Stammbaum, 13 & \\
\hline Frauen, 7 & Ihre, 21 & Nummer, 15 & Student, 11 & \\
\hline
\end{tabular}

Table 10: List of trisyllabic and polysyllabic words in Wir Kids 1

\begin{tabular}{|l|l|l|}
\hline Trisyllabic words & Polysylabbic words \\
\hline Charakter, 22 & Interview, 22 & entschuldigen, 21 \\
\hline Düsseldorf, 11 & langweilig, 19 & extravagant, 24 \\
\hline Einzelkind, 18 & Lehrerin, 21 & Familie, 12 \\
\hline Geschwister, 15 & sympathisch, 19 & Telefonnummer, 20 \\
\hline gewonnen, 8 & Telefon, 21 & verheiratet, 21 \\
\hline Großeltern, 15 & Sum: 11 words & Wiedersehen, 9 \\
\hline & & Sum: 6 words \\
\hline
\end{tabular}


Sofia P. Tsakalidou

SPELLING TEST FOR LEARNERS WITH DYSLEXIA

LEARNING GERMAN AS A SECOND FOREIGN LANGUAGE

\begin{tabular}{|c|c|}
\hline \multicolumn{2}{|r|}{ Table 11: Grapheme categories in Paul, Lisa \& Co. 1} \\
\hline Graphemes & Words \\
\hline$<\mathrm{ei}>$ & nein, drei, heiße, heißt, dreizehn, reiten, zwei, ein, eins, einen, reinkommen, keine \\
\hline$<\mathrm{eu}>$ & Deutsch, neu, neun, neunzehn, heute \\
\hline$<\mathrm{ie}>$ & sieben, spiele, vier, siebzehn, Spiel, sie, Klavier, Geografie, wie, die, hier, vierzehn \\
\hline$<\mathrm{au}>$ & Auto, Frau, Hausaufgaben, Modellauto, schau, faulenzen, auch, aus \\
\hline$<\ddot{\mathrm{a}}>$ & spät \\
\hline$<\ddot{\mathrm{O}}>$ & blöd, zwölf, möchte \\
\hline$<\ddot{u}>$ & Müsli, fünf, fünfzehn \\
\hline$<\mathrm{sch}>$ & Deutsch, Schokolade, Schuh, schau, schon, schwimmen \\
\hline$<\mathrm{sp}>$ & spiele, Spiel, spät \\
\hline$<\mathrm{st}>$ & Stadion \\
\hline$<\mathrm{ch}>$ (ich) & ich, echt, sechzehn, möchte, möchten, möchtest \\
\hline$<\mathrm{ch}>$ (machen) & acht, mache, machst, suche, doch, Buch, achtzehn, Nachmittag \\
\hline$<\mathrm{ck}>$ & Socke \\
\hline$<\mathrm{ph}>$ & Alphabet, Physik \\
\hline$<\mathrm{er}>$ & Bruder, woher, wer, der, hier, Computer, super \\
\hline$<$ th $>$ & Mathematik \\
\hline$<\mathrm{g}>$ & lustig, zwanzig \\
\hline$<\mathrm{d}>$ & Bild \\
\hline$<$ chs $>$ & sechs \\
\hline Sum: & 83 words \\
\hline
\end{tabular}

Table 12: Grapheme categories in Luftballons

\begin{tabular}{|c|l|}
\hline Graphemes & \multicolumn{1}{|c|}{ Words } \\
\hline$<$ ei $>$ & bei, dein, Eis, heißt, mein, nein, zwei, heiße, heißen, meine, Österreich \\
\hline$<\mathrm{eu}>$ & Deutsch, deutsche, Deutschland, Europa \\
\hline$<\mathrm{e}>$ & $\begin{array}{l}\text { die, wie, Liebe, spiele, griechische, Wiedersehen, Biologie, Familienname, } \\
\text { Radiergummi }\end{array}$ \\
\hline$<\mathrm{au}>$ & auch, auf, Frau, Maus, Umlaut \\
\hline$<$ äu $>$ & Mäuschen, häufige \\
\hline$<$ ä $>$ & März, Bärchen, Dänemark \\
\hline$<$ ö $>$ & zwölf, Österreich \\
\hline$<$ ü $>$ & fünf, grün, grüßt, Menü, müde, München, Tschüss \\
\hline$<$ sch $>$ & Deutsch, Schatz, Tschüss, deutsche, Deutschland, griechische \\
\hline$<$ sp $>$ & Sportlehrer, spiele \\
\hline$<$ st $>$ & Buchstabe \\
\hline$<\mathrm{ch}>$ (ich) & $\begin{array}{l}\text { ich, mich, Milch, nicht, Bärchen, München, Engelchen, griechische, Österreich, } \\
\text { Mäuschen }\end{array}$ \\
\hline$<\mathrm{ch}>$ (machen) & Nacht, Buchstabe, auch \\
\hline$<\mathrm{qu}>$ & Quelle \\
\hline$<$ ph $>$ & Alphabet, Xylophon \\
\hline$<\mathrm{er}>$ & der, Fenster, Kinder, Computer, Sportlehrer, Wiedersehen \\
\hline$<\mathrm{g}>$ & Tag, Zug \\
\hline$<\mathrm{d}>$ & Abend \\
\hline Sum: & 77 words \\
\hline
\end{tabular}




\begin{tabular}{|c|c|}
\hline \multicolumn{2}{|r|}{ Table 13: Grapheme categories in Wir Kids 1} \\
\hline Graphemes & Words \\
\hline$<\mathrm{ei}>$ & $\begin{array}{l}\text { Einzelkind, verheiratet, dein, deine, dreißig, dreizehn, einen, langweilig, drei, eins, } \\
\text { heiße, heißen, heißt, mein, keine, mein, meine, nein, Schweiz, zwei }\end{array}$ \\
\hline$<\mathrm{eu}>$ & deutsch, Freundin, freundlich, Freund, Leute, neun, neunzehn, neunzig \\
\hline$<\mathrm{ie}>$ & $\begin{array}{l}\text { Familie, Wiedersehen, die, sie, Spiel, sieben, vier, siebzehn, siebzig, Wien, Spiele, wie, } \\
\text { viele, vierzehn, vierzig }\end{array}$ \\
\hline$<\mathrm{au}>$ & auf, aus, Frauen, Frau \\
\hline$<\ddot{a}>$ & Mädchen \\
\hline$<\ddot{0}>$ & blöd, zwölf \\
\hline$<\ddot{\mathrm{u}}>$ & Düsseldorf, Brüder, fünfzehn, fünfzig, Grüße, grüßen, fünf, für, grüßt, Tschüs, Würfel \\
\hline$<\mathrm{sch}>$ & entschuldigen, Geschwister, deutsch, Mensch, Schweiz, Tschüs, Schwester, Schwestern \\
\hline$<\mathrm{sp}>$ & Spiel, Spiele \\
\hline$<$ st $>$ & streng, Stammbaum, Student, Stuttgart \\
\hline$<\mathrm{ch}>$ (ich) & dich, ich, Mädchen, nicht, sechzehn, sechzig \\
\hline $\begin{array}{c}<\mathrm{ch}> \\
\text { (machen) }\end{array}$ & acht, achtzehn, achtzig, Tochter \\
\hline$<e r>$ & $\begin{array}{l}\text { Charakter, Alter, Bruder, Brüder, Geschwister, Interview, Telefonnummer, verheiratet, } \\
\text { der, hundert, Herr, Kinder, Mutter, Nummer, wer, Tochter, Vater }\end{array}$ \\
\hline$<$ th $>$ & sympathisch \\
\hline$<\mathrm{g}>$ & $\begin{array}{l}\text { achtzig, dreißig, langweilig, fünfzig, lustig, neunzig, Tag, sechzig, siebzig, vierzig, } \\
\text { zwanzig }\end{array}$ \\
\hline$<\mathrm{d}>$ & sind \\
\hline$<$ chs $>$ & sechs \\
\hline$<\mathrm{ng}>$ & singen \\
\hline Sum: & 117 words \\
\hline
\end{tabular}

We also recorded some international words, which were found in the three books (for example Adresse, Alphabet, Name, Ball, Musik, Foto) or were commonly used (for example Delfin, Fan, Geografie, Karte). Consequently, we compared the words of the three books (Tables 14-17) and classified them into four categories: (a) irregular words common in all books (CIW, common irregular words) (19 words), (b) irregular international words (IIW) (25 words), (c) regular words common in all books (CRW, common regular words) (12 words) and (d) regular international words (RIW) (37 words). The total number of words was 93 and we used 30 of them for the Spelling Test. 
Table 14: Selected words category CIW (common irregular words), number of syllables and dipthongs/consonant clusters

\begin{tabular}{|l|c|c|c|l|c|c|c|}
\hline Word & Category & $\begin{array}{c}\text { Number } \\
\text { of } \\
\text { syllables }\end{array}$ & $\begin{array}{c}\text { Dipthong/ } \\
\text { consonant } \\
\text { cluster }\end{array}$ & Word & Category & $\begin{array}{c}\text { Number } \\
\text { of } \\
\text { syllables }\end{array}$ & $\begin{array}{c}\text { Dipthong/ } \\
\text { consonant } \\
\text { cluster }\end{array}$ \\
\hline spielt & CIW & 1 & ie & ein & CIW & 1 & ei \\
\hline heißt & CIW & 1 & ei & zwölf & CIW & 1 & ö \\
\hline sie & CIW & 1 & ie & Frau & CIW & 1 & au \\
\hline Pferd & CIW & 1 & pf & fünf & CIW & 1 & ü \\
\hline nein & CIW & 1 & ei & zwei & CIW & 1 & ei \\
\hline neun & CIW & 1 & eu & vier & CIW & 1 & ie, -er \\
\hline die & CIW & 1 & ie & Deutsch & CIW & 1 & eu, sch \\
\hline wie & CIW & 1 & ie & heiße & CIW & 2 & ei \\
\hline ich & CIW & 1 & ch (ich) & spiele & CIW & 2 & ie, sp \\
\hline der & CIW & 1 & -er & \multicolumn{2}{|l}{ Sum: } & $\mathbf{1 9}$ words \\
\hline
\end{tabular}

Table 15: Selected words category IIW (irregular international words), number of syllables and dipthongs/consonant clusters

\begin{tabular}{|c|c|c|c|c|c|c|c|}
\hline Word & Category & $\begin{array}{c}\text { Number } \\
\text { of } \\
\text { syllables }\end{array}$ & $\begin{array}{c}\text { Dipthong/ } \\
\text { consonant } \\
\text { cluster }\end{array}$ & Word & Category & $\begin{array}{c}\text { Number } \\
\text { of } \\
\text { syllables }\end{array}$ & $\begin{array}{c}\text { Dipthong/ } \\
\text { consonant } \\
\text { cluster }\end{array}$ \\
\hline Maus & IIW & 1 & $\mathrm{au}$ & Physik & IIW & 2 & ph \\
\hline Sport & IIW & 1 & $\mathrm{sp}$ & Müsli & IIW & 2 & $\ddot{\mathrm{u}}$ \\
\hline Buch & IIW & 1 & $\mathrm{ch}$ & Interview & IIW & 3 & - \\
\hline Schuh & IIW & 1 & sch & Schokolade & IIW & 4 & sch \\
\hline singt & IIW & 1 & ng & Xylophon & IIW & 3 & ph \\
\hline Freund & IIW & 1 & eu & Alphabet & IIW & 3 & ph \\
\hline singen & IIW & 2 & ng & Computer & IIW & 3 & -er \\
\hline Menü & IIW & 2 & $\ddot{\mathrm{u}}$ & Charakter & IIW & 3 & ch, -er \\
\hline Kinder & IIW & 2 & -er & Mathematik & IIW & 4 & th \\
\hline super & IIW & 2 & -er & Familie & IIW & 4 & ie \\
\hline Nummer & IIW & 2 & -er & Biologie & IIW & 4 & ie \\
\hline Stadion & IIW & 2 & st & Geografie & IIW & 4 & ie \\
\hline Student & IIW & 2 & st & & & Sum: & 25 words \\
\hline
\end{tabular}

Table 16: Selected words category CRW (common regular words), number of syllables and dipthongs/consonant clusters

\begin{tabular}{|l|c|c|c|l|c|c|c|}
\hline Word & Category & $\begin{array}{c}\text { Number } \\
\text { of } \\
\text { syllables }\end{array}$ & $\begin{array}{c}\text { Dipthong/ } \\
\text { consonant } \\
\text { cluster }\end{array}$ & Word & Category & $\begin{array}{c}\text { Number } \\
\text { of } \\
\text { syllables }\end{array}$ & $\begin{array}{c}\text { Dipthong/ } \\
\text { consonant } \\
\text { cluster }\end{array}$ \\
\hline bin & CRW & 1 & - & ist & CRW & 1 & - \\
\hline das & CRW & 1 & - & und & CRW & 1 & - \\
\hline du & CRW & 1 & - & gute & CRW & 2 & - \\
\hline ja & CRW & 1 & - & guten & CRW & 2 & - \\
\hline von & CRW & 1 & - & hallo & CRW & 2 & - \\
\hline bist & CRW & 1 & - & Name & CRW & 2 & - \\
\hline \multicolumn{7}{|l|}{} \\
\hline
\end{tabular}


Table 17: Selected words category RIW (regular international words), number of syllables and dipthongs/consonant clusters

\begin{tabular}{|l|c|c|c|l|c|c|c|}
\hline Word & Category & $\begin{array}{c}\text { Number } \\
\text { of } \\
\text { syllables }\end{array}$ & $\begin{array}{c}\text { Dipthong/ } \\
\text { consonant } \\
\text { cluster }\end{array}$ & \multicolumn{1}{|c|}{ Word } & Category & $\begin{array}{c}\text { Number } \\
\text { of } \\
\text { syllables }\end{array}$ & $\begin{array}{c}\text { Dipthong/ } \\
\text { consonant } \\
\text { cluster }\end{array}$ \\
\hline Ball & RIW & 1 & - & Karte & RIW & 2 & - \\
\hline Bus & RIW & 1 & - & Klasse & RIW & 2 & - \\
\hline Fan & RIW & 1 & - & Lampe & RIW & 2 & - \\
\hline Film & RIW & 1 & - & skaten & RIW & 2 & - \\
\hline Mai & RIW & 1 & - & Party & RIW & 2 & - \\
\hline im & RIW & 1 & - & Fußball & RIW & 2 & - \\
\hline Clown & RIW & 1 & - & Maria & RIW & 3 & - \\
\hline Foto & RIW & 2 & - & ABC & RIW & 3 & - \\
\hline Fotos & RIW & 2 & - & beginnen & RIW & 3 & - \\
\hline Hobby & RIW & 2 & - & Telefon & RIW & 3 & - \\
\hline Idee & RIW & 2 & - & Volleyball & RIW & 3 & - \\
\hline Kino & RIW & 2 & - & Elefant & RIW & 3 & - \\
\hline lila & RIW & 2 & - & Alexis & RIW & 3 & - \\
\hline Papa & RIW & 2 & - & Adresse & RIW & 3 & - \\
\hline Mama & RIW & 2 & - & Ypsilon & RIW & 3 & - \\
\hline Musik & RIW & 2 & - & Februar & RIW & 3 & - \\
\hline Note & RIW & 2 & - & Basketball & RIW & 3 & - \\
\hline Tennis & RIW & 2 & - & Marmelade & RIW & 4 & - \\
\hline Globus & RIW & 2 & - & & & Sum: & 37 words \\
\hline
\end{tabular}

The Spelling Test is a tool used to record specific spelling difficulties of learners, in order to plan and organize the intervention and differentiate instruction. During the research it was administered to all learners with and without dyslexia. It consists of many spelling patterns, as well as exceptions to spelling rules, nouns, verbs, conjunctions and adverbs. The types of spelling errors examined are: Grammatical errors (Gr1), Phonological errors (Ph1-Ph14), and Other errors (O1-O6). The Spelling Test started with very simple words, consisted of 30 words, had a maximum number of points (30 points) and every word, which was spelled correctly equals one point.

The original test (Mouzaki, Protopapas, Sideridis, \& Simos, 2010) was designed with an interruption rule. After six misspelled words in a row, the test would stop. This interruption rule was also performed in the Spelling Test for the German language. Although, due to the fact that it was administered inside the classroom to all the learners simultaneously, the interruption rule was taken into consideration, while correcting the test and not while administering it. This decision was made for two reasons. The first reason was so that there were no feelings of failure for the learners, who didn't manage to write many answers correctly. The second reason was, that it was quite time consuming to administer this test individually, due to the fact that there was a variety of tools used in this research and there was not the commodity of time. 


\section{Results and Discussion}

Two levels of analysis were performed after the data was gathered: (a) We counted the sum of points (i.e., words spelled correctly) and compared the two populations (learners with and without dyslexia). (b) We analysed the errors all the learners with dyslexia did in detail, in order to understand their more common difficulties. We used two tools for this analysis, namely a synoptic table of spelling errors (Table 18) and a detailed table of spelling errors (Table 19).

Table 18: Synoptic table of spelling errors (example)

\begin{tabular}{|c|c|c|c|c|c|c|c|c|c|c|c|c|}
\hline \multirow{2}{*}{\multicolumn{2}{|c|}{ Word }} & \multirow{3}{*}{ 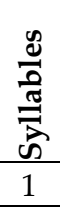 } & \multirow{3}{*}{ 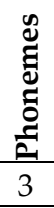 } & \multicolumn{6}{|c|}{ Phonological errors } & \multicolumn{3}{|c|}{ Other errors } \\
\hline & & & & \multirow[t]{2}{*}{ בี } & \multirow{2}{*}{$\frac{\stackrel{d}{E}}{1}$} & \multirow[t]{2}{*}{$\stackrel{\mathscr{L}}{\underline{L}}$} & \multirow{2}{*}{$\frac{\frac{9}{1}}{\frac{1}{1}}$} & \multirow[t]{2}{*}{$\frac{\mathfrak{Z}}{\mathfrak{E}}$} & \multirow[t]{2}{*}{$\frac{\Xi}{I}$} & \multirow[t]{2}{*}{5} & \multirow[t]{2}{*}{ ธิ } & \multirow[t]{2}{*}{0} \\
\hline 5 & aus & & & & & & & & & & & \\
\hline 6 & sie & 1 & 2 & 1 & & & ie & & & & & \\
\hline 7 & zwei & 1 & 4 & 1 & & & ei & $\mathrm{zW}$ & & & & \\
\hline 8 & neun & 1 & 4 & & & & eu & & & & & \\
\hline 9 & heißt & 1 & 5 & 1 & & & ei & $\beta \mathrm{t}$ & & & & \\
\hline 10 & zwölf & 1 & 5 & & & & & $z W$, lf & & 1 & & \\
\hline 11 & Deutsch & 1 & 5 & & & & eu & & (t)sch & & & \\
\hline 12 & Foto & 2 & 4 & & & & & & & & & 1 \\
\hline 13 & Musik & 2 & 5 & & & & & & & & & 1 \\
\hline 15 & komme & 2 & 4 & & & & & $\mathrm{~mm}$ & & & & \\
\hline 16 & sieben & 2 & 5 & 1 & & & ie & & & & & \\
\hline 17 & Jahre & 2 & 4 & 1 & & & & hr & & & & \\
\hline 18 & spiele & 2 & 5 & & & & ie & $\mathrm{sp}$ & & & & \\
\hline 19 & fünfzehn & 2 & 7 & 2 & & 1 & & $\mathrm{nf}, \mathrm{hn}$ & & & & \\
\hline 20 & nein & 1 & 4 & & & & ei & & & & & \\
\hline 21 & Lampe & 2 & 5 & & & & & $\mathrm{mp}$ & & & & 1 \\
\hline 22 & Fußball & 2 & 6 & 3 & & & & $\mathrm{Bb}, \mathrm{ll}$ & & & & \\
\hline 23 & dreizehn & 2 & 7 & 1 & & 1 & ei & $\mathrm{dr}, \mathrm{hn}$ & & & & \\
\hline 24 & Telefon & 3 & 7 & & & & & & & & & 1 \\
\hline 25 & Elefant & 3 & 7 & 1 & & & & $\mathrm{nt}$ & & & & 1 \\
\hline 26 & Basketball & 3 & 9 & & & & & sk, tb, 11 & & & & 1 \\
\hline 27 & Griechenland & 3 & 10 & & & & ie & Gr, ch, nl, nd & & 1 & & \\
\hline 28 & Marmelade & 4 & 9 & 1 & & 1 & & & & & & 1 \\
\hline 29 & Hausaufgaben & 4 & 12 & & & & $\mathrm{au}, \mathrm{au}$ & $\mathrm{fg}$ & & & 1 & \\
\hline 30 & Deutschland & 2 & 9 & 2 & & 2 & eu & nd & (t)sch & & & \\
\hline \multicolumn{2}{|c|}{ Learner's sum } & & & 15 & 1 & 5 & 10 & 2 & 1 & 2 & 1 & 7 \\
\hline \multicolumn{2}{|c|}{ Total sum } & 56 & & \multicolumn{2}{|c|}{157} & & 13 & 29 & 2 & \multicolumn{2}{|c|}{30} & 179 \\
\hline
\end{tabular}

In the synoptic table of spelling errors (Table 18) we noted the error type(s) of all words written incorrectly, as well as the sum of every error type. In the detailed table of spelling errors (Table 19) we noted all words written incorrectly for every error type. In this table we, also, gave the word written incorrectly and the correct word in brackets. Furthermore, the numeric data in this table are the number of errors, the chances of each particular error in this Spelling Test and the ratio (\%) of every error type. 
Table 19: Detailed table of spelling errors (example)

\begin{tabular}{|c|c|c|c|c|c|}
\hline Code & Error type & Example & $\begin{array}{c}\text { Number of } \\
\text { errors }\end{array}$ & $\begin{array}{c}\text { Chances of } \\
\text { error }\end{array}$ & $\begin{array}{l}\text { Ratio \% } \\
\text { (f \%) }\end{array}$ \\
\hline Gr & \multicolumn{5}{|c|}{ Grammatical errors } \\
\hline Gr1 & $\begin{array}{l}\text { Inflectional ending } \\
\text { (verbs) }\end{array}$ & heißs (heißt) & 1 & 4 & $25 \%$ \\
\hline $\mathbf{P h}$ & \multicolumn{5}{|c|}{ Phonological errors } \\
\hline $\mathrm{Ph} 2$ & $\begin{array}{l}\text { Phoneme } \\
\text { substitution }\end{array}$ & $\begin{array}{l}\text { wie (sie), heißs (heißt), chvolf (zwölf), } \\
\text { come (komme), sieden (sieben), giare } \\
\text { (Jahre), Fusßall (Fußball), draitsen } \\
\text { (dreizehn), Grexelant (Griechenland), } \\
\text { Marmelabe (Marmelade), } \\
\text { houseafugaden (Hausaufgaben) }\end{array}$ & 14 & 157 & $8,9 \%$ \\
\hline $\mathrm{Ph} 4$ & Adding a phoneme & $\begin{array}{l}\text { giare (Jahre), founf (fünfzehn), } \\
\text { Fusßall (Fußball), houseafugaden } \\
\text { (Hausaufgaben) }\end{array}$ & 4 & 157 & $2,5 \%$ \\
\hline $\mathrm{Ph} 5$ & Omitting a syllable & founf (fünfzehn) & 1 & 56 & $1,8 \%$ \\
\hline $\mathrm{Ph} 6$ & Omitting a phoneme & $\begin{array}{l}\text { heißs (heißt), Grexelant } \\
\text { (Griechenland) }\end{array}$ & 2 & 157 & $1,3 \%$ \\
\hline $\mathrm{Ph} 9$ & $\begin{array}{l}\text { Reversing } \\
\text { diphthongs }\end{array}$ & $\begin{array}{l}\text { zwie (zwei), houseafugaden } \\
\text { (Hausaufgaben) }\end{array}$ & 2 & 13 & $15,4 \%$ \\
\hline Ph10 & $\begin{array}{l}\text { Diphthong } \\
\text { simplification }\end{array}$ & $\begin{array}{l}\text { Doits (Deutsch), draitsen (dreizehn), } \\
\text { Grexelant (Griechenland), Doitsland } \\
\text { (Deutschland) }\end{array}$ & 4 & 13 & $30,8 \%$ \\
\hline Ph12 & $\begin{array}{l}\text { Simple consonant } \\
\text { cluster simplification }\end{array}$ & $\begin{array}{l}\text { come (komme), giare (Jahre), draitsen } \\
\text { (dreizehn), Grexelant (Griechenland) }\end{array}$ & 4 & 29 & $13,8 \%$ \\
\hline Ph14 & $\begin{array}{l}\text { Simplification of } \\
\text { consonant cluster } \\
\text { with } 3 \text { or } 4 \text { letters } \\
\end{array}$ & $\begin{array}{l}\text { Doits (Deutsch), Doitsland } \\
\text { (Deutschland) }\end{array}$ & 2 & 2 & $100 \%$ \\
\hline $\mathbf{O}$ & \multicolumn{5}{|c|}{ Other errors } \\
\hline $\mathrm{O} 2$ & Replacing word & out (und), oush (aus) & 2 & 30 & $6,7 \%$ \\
\hline O5 & $\begin{array}{l}\text { Substitution of } \\
\text { uppercase/lowercase } \\
\text { letters }\end{array}$ & $\begin{array}{l}\text { musik (Musik), giare (Jahre), lampe } \\
\text { (Lampe), telefon (Telefon), } \\
\text { houseafugaden (Hausaufgaben) }\end{array}$ & 5 & 179 & $2,8 \%$ \\
\hline $\mathrm{O} 6$ & Omitting Umlaut & chvolf (zwölf), founf (fünfzehn) & 2 & 2 & $100 \%$ \\
\hline
\end{tabular}

\section{Recommendations}

The Spelling Test presented in this article in detail is the first effort to construct a test in order to record the specific spelling difficulties learners face, when learning German as a second foreign language. This test is suitable for learners with and without dyslexia and could be used for every student. Through this test and the proposed error type analysis, the teacher can have detailed information as to the most common errors each learner makes. This information can help the teacher organize and plan his intervention and possible teaching method modifications in the classroom (if considered necessary).

In this way, every learner is respectfully considered as a unique person, with his/her own difficulties and learning needs. 


\title{
6. Conclusion
}

This tool could be used for spelling evaluation in the first stages of learning German as a second foreign language, in order to record the specific difficulties every learner faces and be able to attend to his/her needs. The Spelling Test presented could have an impact and use in the scientific field as well, as it could be used for larger scale research. It could also be used as a fundament, in order to create a Spelling Test for other reference levels as well. Furthermore, it could be used by teachers and researchers of other foreign languages, who would like to construct a Spelling Test as well.

As this specific Spelling Test was used in order to identify spelling difficulties of all learners (in particular learners with dyslexia), the specific error type categorization helped the teacher organize and plan intervention and teaching methodology modifications, which were adapted during Phase 2. Also, the use of the Spelling Test during Phase 3 helped the researcher estimate the post-intervention performance of all learners (with and without dyslexia).

In conclusion, we should notice, that our education system does not help teachers adjust to all individual needs in their classroom. There is a "one size fits all" policy (Bondie, Dahnke \& Zusho, 2019), which is far from the truth of every day teaching. Teachers face mixed ability classrooms every day and learners with dyslexia, as well as other specific learning difficulties are always included. If a teacher can have a clear image of the specific difficulties in spelling, for example, he will be able to adapt the lesson to the individual needs of all learners.

\section{Conflict of Interest Statement}

The author declares no conflicts of interests.

\section{Acknowledgement}

The author certifies that she has no commercial associations (eg, consultancies, stock ownership, equity interest, patent/licensing arrangements, etc) that might pose a conflict of interest in connection with the submitted article.

\begin{abstract}
About the Author
Sofia P. Tsakalidou completed her PhD in Didactics of German as a Second foreign language at Aristotle University of Thessaloniki. In her $\mathrm{PhD}$ research she examines teaching spelling to learners with dyslexia and focuses on developing assessment tools as well as applying teaching modifications and intervention. She is currently conducting her postdoctoral research at the Department of German Language and Literature at Aristotle University of Thessaloniki. Her research focuses on the field of special education and especially students with specific learning disabilities, including studies on the nature and the assessment of learning disabilities of children in German language. Her interests focus on teacher training as to the subject of her doctoral and postdoctoral research, namely learners with dyslexia and diversity in the classroom in general. Furthermore,
\end{abstract}


she has worked as an EFL and a GFL teacher for 20 years in private and public schools in both Primary and Secondary education.

\section{References}

Andreou, G., \& Baseki, J. (2012). Phonological and Spelling Mistakes among Dyslexic and Non-Dyslexic Children Learning Two Different Languages: Greek vs English. Psychology, 3(8), 595-600. doi: 10.4236/psych.2012.38089

Bahr, R. H., Silliman, E. R., \& Berninger, V. W. (2009). What spelling errors have to tell about vocabulary learning. In C. Wood, \& V. Connelly (Eds.). Reading and Spelling: Contemporary perspectives (pp. 109-129). New York: Routledge.

Bondie, R. S., Dahnke, C., \& Zusho, A. (2019). How does changing ,One-size-fits-all' to differentiated instruction affect teaching? Review of Research in Education, 43, 336362. doi: 10.3102/0091732X18821130

Bovermann, M., Georgiakaki, M., \& Zschärlich, R. (2013). Paul, Lisa \& Co für die Grundschule. Kurs- E Arbeitsbuch. Athen: Hueber Hellas.

Jenkins-Krumm, E. M. \& Clement, C. (2014). Wir Kids A1.1. Grundkurs Deutsch für junge Lernende. Lehr- und Arbeitsbuch mit Audio-CD. Athen: Klett Verlag GmbH.

Kounalaki, M., Delikeisoglou, A., Brodowski-Frogaki, A., Koutentaki, M., \& Papandreou, N. (2011). Luftballons. Lehr- und Arbeitsbuch. Kids A. Neu. Crete: Kounalaki Verlag.

Landerl, K., Wimmer, H., \& Moser, E. (1997). SLRT - Salzburger Lese- und Rechtschreibtest. Bern: Hans Huber.

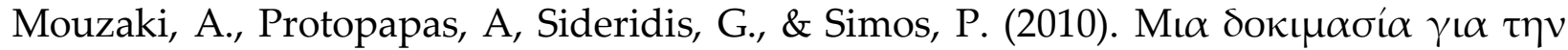

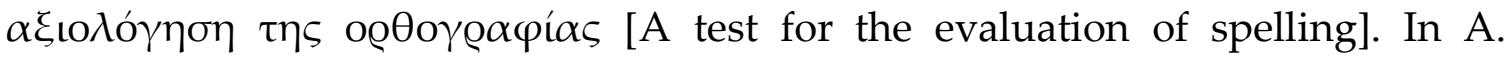

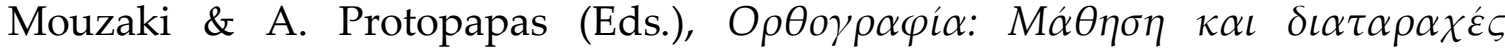
[Spelling: learning and disorders] (pp. 326-347). Athens: Gutenberg.

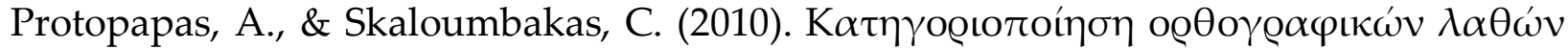
[Classification of spelling errors]. In A. Mouzaki \& A. Protopapas (Eds.),

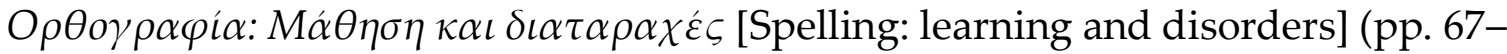
104). Athens: Gutenberg.

Reraki, M. (2020). Chapter ten. Dyslexia-friendly teaching in Greek EFL classrooms. In F. Anastassiou \& G. Andreou (Eds.). English as a foreign language. Perspectives on teaching, multilingualism and interculturalism (pp. 211-237). Newcastle upon Tyne: Cambridge Scholars Publishing.

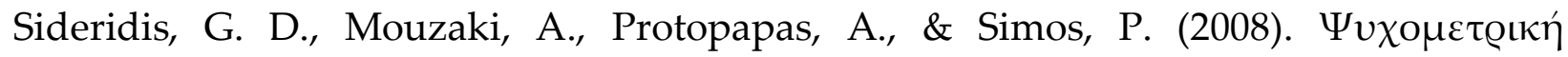

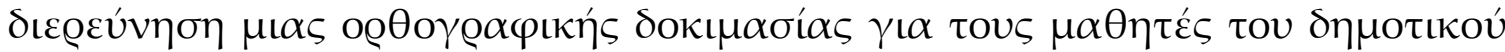
$\sigma \chi 0 \lambda \varepsilon$ cíov [Psychometric evaluation of a spelling test for elementary school students]. Psychologia, 15, 290-315.

Sougari, A. M., \& Mavroudi, A. (2019). Differentiated instruction in the EFL classroom: Discrepancies between teacher's self-report questionnaires and actual practices. In Selected Papers of ISTAL 2019, 398-413. 
Stock, C., \& Schneider, W. (2007). DERET 1-2+. Deutscher Rechtschreibtest für das erste und zweite Schuljahr. Göttingen: Hogrefe.

Talli, I. (2010). Linguistic abilities in developmental dyslexia and specific language impairment (SLI): a comparative and cross-linguistic approach. (Unpublished Doctoral Dissertation). L' Université Paris Descartes \& Aristotle University of Thessaloniki, Thessaloniki.

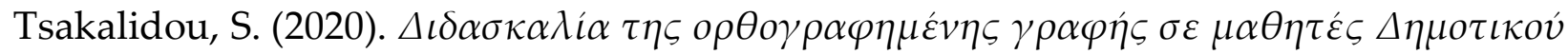

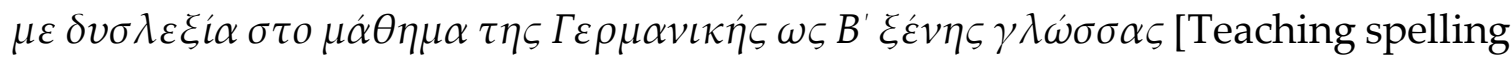
to learners with dyslexia learning German as a second Foreign Language in primary education] (Unpublished doctoral dissertation). Department of German Language and Literature, Aristotle University of Thessaloniki, Thessaloniki.

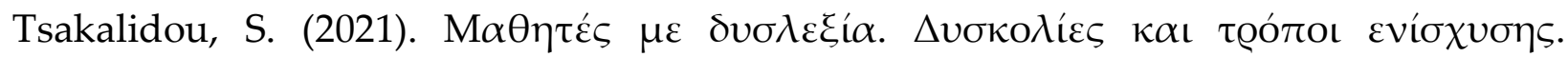
[Learners with dyslexia. Difficulties and ways to support them.] Aktuell, 52, 16-19.

Tsakalidou, S. (in press). Effectiveness of spelling interventions for learners with dyslexia learning German as a second foreign language. ICOMEU 2020. Proceedings of $3^{\text {rd }}$ International Conference on Management of Educational Units (18-20/12/2020). Preparing for new realities in Education: Opportunities and Challenges. International Hellenic University.

Tsakalidou, S. (in press). Ohns Flsiß ksin Prsis. $\Delta \iota \delta \alpha ́ \alpha \sigma o v \tau \alpha \varsigma \Gamma \varepsilon \rho \mu \alpha v \iota \kappa \alpha ́ \alpha \sigma \varepsilon \mu \alpha \theta \eta \tau \dot{\varepsilon} \varsigma \mu \varepsilon$ $\delta v \sigma \lambda \varepsilon \xi i \alpha$ [Teaching German to learners with dyslexia]. Athen: Karabatos Hueber Hellas Verlag.

Tsakalidou, S., Koufokotsiou, P., \& Gaganis, C. (in press). Legasthenie und Binnendifferenzierung im DaF-Unterricht. In: E. Karagiannidou, C.O. Papadopoulou, \& R. M. Sidiropoulou (Hg.), Verbindung von Theorie und Praxis in der DaF-Lehrerausbildung: Praktikumsprogramm und Vorträge der 1. bis 7. DaFDidaktik-Tagung in Thessaloniki. Festschrift für Aikaterini Vretta-Panidou. Thessaloniki: University Studio Press. 
Authors will retain the copyright of their published articles agreeing that a Creative Commons Attribution 4.0 International License (CC BY 4.0) terms wil be applied to their work. Under the terms of this license, no permission is required from the author(s) or publisher for members of the community to copy, distribute, transmit or adapt the article content, providing a proper, prominent and unambiguous attribution to the authors in a manner that makes clear that the materials are being reused under permission of a Creative Commons License. Views, opinions and conclusions expressed in this research article are views, opinions and conclusions of the author(s). Open Access Publishing Group and European Journal of Special Education Research shall not be responsible or answerable for any loss, damage or liability caused in relation to/arising out of conflict of interests, copyright violations and inappropriate or inaccurate use of any kind content related or integrated on the research work. All the published works are meeting the Open Access Publishing requirements and can be freely accessed, shared, modified, distributed and used in educational, commercial and non-commercial purposes under a Creative Commons Attribution 4.0 International License (CC BY 4.0). 